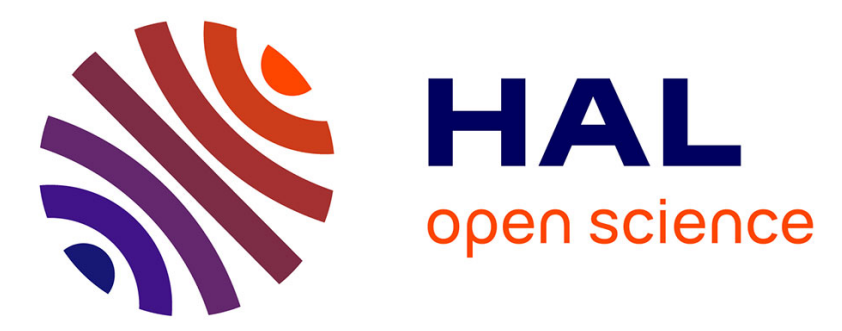

\title{
Control of the incubation time in the vapor-solid-solid growth of semiconductor nanowires
}

M. Orrù, M den Hertog, Eric Robin, Y. Genuist, Régis André, Joel Cibert, Edith Bellet-Amalric

\section{- To cite this version:}

M. Orrù, M den Hertog, Eric Robin, Y. Genuist, Régis André, et al.. Control of the incubation time in the vapor-solid-solid growth of semiconductor nanowires. Applied Physics Letters, 2017, 110 (26), pp.263107. 10.1063/1.4985713 . hal-01583052

\section{HAL Id: hal-01583052 \\ https://hal.science/hal-01583052}

Submitted on 6 Sep 2017

HAL is a multi-disciplinary open access archive for the deposit and dissemination of scientific research documents, whether they are published or not. The documents may come from teaching and research institutions in France or abroad, or from public or private research centers.
L'archive ouverte pluridisciplinaire HAL, est destinée au dépôt et à la diffusion de documents scientifiques de niveau recherche, publiés ou non, émanant des établissements d'enseignement et de recherche français ou étrangers, des laboratoires publics ou privés. 
A

A Control of the incubation time in the vapor-solid-solid growth of

\section{Publisfmic onductor nanowires}

M. Orrù, ${ }^{1,2,3, a)}$ M. den Hertog, ${ }^{1,3}$ E. Robin, ${ }^{1,2}$ Y. Genuist, ${ }^{1,3}$ R. André, ${ }^{1,3}$ J. Cibert, ${ }^{1,3}$ and E.

Bellet-Amalric ${ }^{1,2}$

1) Univ. Grenoble Alpes, F-38000 Grenoble, France

2) CEA, INAC, F-38000 Grenoble, France

3) CNRS, Institut Néel, F-38000 Grenoble, France

(Dated: 1 June 2017)

Nanowires grown in the vapor-solid-solid mode using solid gold nanoparticles as a catalyst may exhibit a strong fluctuation of their length mostly due to the presence of an incubation time with a large distribution. We show that this is efficiently cured by an appropriate preparation of the catalyst nanoparticle - in the case of ZnTe nanowires by adding a Zn flux during the dewetting process. While NWs start at any time after dewetting in vacuum (resulting in a broad length distribution, up to a factor of 10), the incubation time is quite uniform after dewetting under Zn exposure. Residual fluctuations (reduced to below a factor of 2) are due to fluctuations of the nanoparticle size and to a change of the nanoparticle morphology during the growth.

There is a strong interest in the growth of semiconductor nanowires (NWs) for nanophotonics ${ }^{1}$ and quantum technologies. Advanced NW structures embed a quantum dot (QD) with a highly flexible range of characteristics (composition, shape, size). For instance, it is possible to design a QD which features a hole ground state with light-hole character ${ }^{2,3}$, a highly desirable feature in the frame of quantum technologies ${ }^{4}$. The experimental realization of such a QD has been demonstrated recently ${ }^{5}$ using II-VI NWs, which offer the additional opportunity to incorporate magnetic properties by using diluted magnetic semiconductors ${ }^{5,6}$.

NWs are most often grown using a liquid droplet as a catalyst: this is the so-called vapor-liquid-solid (VLS) mode. It was proposed ${ }^{7}$ that sharper interfaces are obtained by using the vapor-solid-solid growth mode (VSS), with a solid nanoparticle as a catalyst. We reported previously on the VSS growth of ZnTe NWs using gold nanoparticles 8,9 . ZnTe NWs with the zincblende crystal structure were shown to present a broad length distribution, up to more than a factor of ten between the shortest and the longest $\mathrm{NW}^{9}$. We observed no apparent correlation with the distribution of the catalyst diameter, instead we could ascribe it to the presence of a long incubation time, different from NW to NW within the same sample.

Incubation times are often observed during the nucleation of semiconductor NWs grown by VLS, such as GaAs NWs ${ }^{10}$ on $\mathrm{Si}$ substrates, $\mathrm{Si} \mathrm{NWs}^{11} \mathrm{Ge} \mathrm{NWs}^{12}$ and $\mathrm{GaN} \mathrm{NWs}^{13}$. A dispersion of this incubation time can have dramatic consequences, such as a broad distribution of the NW length ${ }^{10,11}$ or even different NW morphologies ${ }^{13}$. The presence of parasitic growth (the growth of ill-featured nanostructures ${ }^{10,14}$ ) and conse-

\footnotetext{
a)marta.orru@neel.cnrs.fr
}

quently, the number of vertical NWs, can also be affected. Possible solutions involve a precise adjustment of the growth conditions (e.g., in the autocatalytic growth of GaAs NWs on $\mathrm{Si}^{14}$ ) or a pre-treatment of the initial gold film in order to ensure a uniform saturation of all gold droplets ${ }^{12}$. That such a pre-treatment can be efficient in the case of VSS remains an open question.

- In this letter, we show that the preparation of the $\mathrm{Au}$ nanoparticles is indeed a key parameter to control the incubation time in the VSS growth of ZnTe NWs. We find that exposing the Au catalyst to a $\mathrm{Zn}$ flux during dewetting, improves the yield of vertical NWs from $20 \%$ to $80 \%$. Using CdTe markers, we confirm that all the NWs in the same sample start to grow at the same time. The characteristics of growth are analyzed using the phenomenological model of diffusion-driven growth and the analytical results described in Ref. 9. A statistical analysis of cone-shaped ZnTe NWs grown for a long time and imaged by scanning electron microscopy (SEM) reveals that the remaining length distribution (down to a factor about 2) is due to the dispersion of the catalyst diameter at the beginning of the growth, and to the existence of two different shapes of the catalyst nanoparticle during the growth.

Our standard growth procedure for this study starts with the preparation of a $\mathrm{ZnTe}(111) \mathrm{B}$ buffer layer at $260^{\circ} \mathrm{C}$, on top of a thermally deoxidized GaAs(111)B wafer. Then a sub-monolayer gold film $(0.2 \mathrm{ML})$ is deposited at room temperature. The gold catalysts are formed by dewetting at $350^{\circ} \mathrm{C}$ for $5 \mathrm{~min}$, and during this time the gold film is exposed to a $\mathrm{Zn}$ flux of $0.4 \mathrm{ML} / \mathrm{s}$ ("Zn-dewetting": this is the main difference with respect to "vacuum-dewetting" as in Ref. 9). The NW growth is started at the same temperature by exposing the catalyst to a stoichiometric ZnTe flux of $0.5 \mathrm{ML} / \mathrm{s}$ from a compound solid source effusion cell, for 10 minutes. The reflection high energy electron diffraction (RHEED) pattern indicates that the gold catalysts are crystalline during the entire growth (VSS 
This manuscript was accepted by Appl. Phys. Lett. Click here to see the version of record. 1 grior to growth, fluxes are calibrated by meaPublialikiog, monitoring RHEED intensity oscillations.

The present study describes the results obtained on three series of samples, grown with the following changes made to the standard procedure: series A consists of 6 samples where, during the $5 \mathrm{~min}$ at $350^{\circ} \mathrm{C}$, the gold catalyst was kept in vacuum, or exposed to a Te flux $(0.5 \mathrm{ML} / \mathrm{s})$ or to a $\mathrm{Zn}$ flux $(0.1,0.2,0.4$ and 0.8 $\mathrm{ML} / \mathrm{s}$ ); series B involves a single sample with $5 \mathrm{CdTe}$ markers, 4 embedded in the ZnTe NW and a last one at the end; it was grown at $375^{\circ} \mathrm{C}$ with an additional Te flux ${ }^{9}$ (after $\mathrm{Zn}$-dewetting at $350^{\circ} \mathrm{C}$ and a ramp of $2 \mathrm{~min}$ from 350 to $375^{\circ} \mathrm{C}$ under a $\mathrm{Zn}$ flux of $0.4 \mathrm{ML} / \mathrm{s}$ ). Series $\mathrm{C}$ is made of two samples with a growth time equal to 10 and $40 \mathrm{~min}$, respectively.

All samples were characterized by SEM, with about 100 NWs observed on each sample. In all cases, ZnTe NWs may have on the same sample two different morphologies (cylinder- or cone-shaped) and crystal structures (wurtzite or zinc-blende, respectively) ${ }^{8}$. In addition, the sample of series B was cleaved into a thin slice and isolated NWs were found in the thinner part of the slice. These NWs were observed by scanning transmission electron microscopy (STEM) and geometrical phase analysis (GPA) $)^{15,16}$ was applied to the high resolution images ${ }^{9}$, allowing us to determine the crystal structure and local lattice parameter (hence composition) of these as-grown NWs. Also, energy dispersive $\mathrm{x}$-ray spectroscopy (EDX) was used to characterize NWs transferred onto a holey carbon grid.
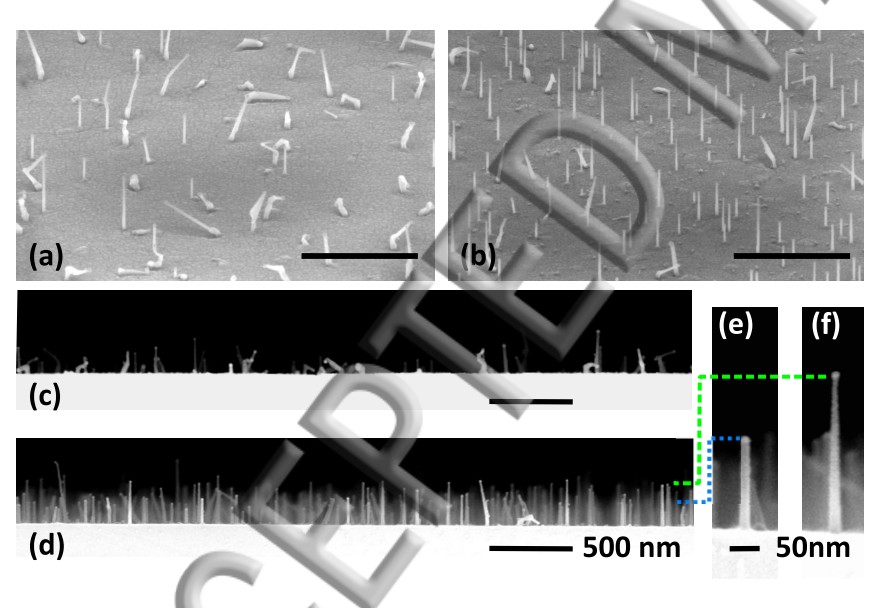

FIG. 1. SEM images of as-grown ZnTe NWs tilted at (a-b) $65^{\circ}$ and (c-d-e-f) $90^{\circ}$. For the sample in panels (a-c) the dewetting was done in vacuum while for the one in (b-d) a $\mathrm{Zn}$ flux of 0.8 $\mathrm{ML} / \mathrm{s}$ was added. In panels (e-f) we show higher magnification images of the two kinds of NWs that are present on the samples, (e) cylinder-shaped with a pyramid at the base and (f) cone-shaped. The height of the cone-(cylinder-) shaped NWs is marked with a green dashed (blue dotted) line. The scale bar for panels (a-b-c-d) is $500 \mathrm{~nm}$.

Series A explores the effect of adding a flux during dewetting. Fig. 1 displays SEM images for two

$\begin{array}{cccc}\text { dewetting } & \text { yield (\%) } & \text { density } / \mu \mathrm{m}^{2} & \text { diameter }(\mathrm{nm}) \\ \text { Zn 0.8 ML/s } & 80 & 13 & 13 \pm 1 \\ \text { Zn 0.4 ML/s } & 72 & 12 & 13 \pm 2 \\ \text { Zn 0.2 ML/s } & 71 & 12 & 14 \pm 2 \\ \text { Zn 0.1 ML/s } & 45 & 5 & 15 \pm 2 \\ \text { vacuum } & 20 & 1 & 18 \pm 2 \\ \text { Te 0.5 ML/s } & 25 & 2 & 17 \pm 2\end{array}$

TABLE I. Summary of results on Series A. From the left, the dewetting conditions, the yield of vertical NWs, the density of vertical NWs, and the average diameter of the gold catalyst (with standard deviation).

samples of the series: dewetting under vacuum as in Ref. 9, or under a Zn flux of $0.8 \mathrm{ML} / \mathrm{s}$. With vacuumdewetting, Fig. 1(a) and (c), parasitic growth takes place and the great majority of objects are not vertical NWs but ill-formed objects. Fig. 1(b)-(d) show that dewetting under $\mathrm{Zn}$ increases the number of vertical NWs (including both cylinder-shaped NWs with a pyramid at their base, Fig. 1(e), and cone-shaped NWs, Fig. 1(f)). The cone-shaped and cylinder-shaped NWs have different average lengths, as evidenced by the green dashed and blue dotted lines, and the length dispersion within each family is small, in contrast to the case of vacuumdewetting 9 . By statistical analysis of SEM images, we measured an improvement of the yield of vertical NWs, from $20 \%$ (vacuum dewetting) to $80 \%$ (dewetting under a Zn flux of $0.8 \mathrm{ML} / \mathrm{s}$ ).

For all the samples of series A, the yield as well as the density of NWs are reported in Table I. Te-dewetting results, at the most, in a moderate improvement, with $25 \%$ of vertical NWs for a Te flux of $0.5 \mathrm{ML} / \mathrm{s}$. The effect of even a small $\mathrm{Zn}$ flux is clear; it somewhat saturates at higher values. Note that the density increases from about 1 to 12 vertical NWs per $\mu \mathrm{m}^{2}$. This is due for a part to the elimination of most parasitic growth, but also to the increase of the total number of objects on the sample, by a factor of about 3 . Such an increase is expected, if the nanoparticle shape is preserved, from the slight decrease of the diameter of the gold nanoparticles, see Table I, from an average of $18 \mathrm{~nm}$ (vacuum dewetting) to $13 \mathrm{~nm}$ (Zn flux of $0.8 \mathrm{ML} / \mathrm{s}$ ).

For the sample of series B, we used the CdTe marker technique $^{18}$ as done in Ref. 9. The crystal structure of the NWs is determined by indexing the Fourier Transform of the high resolution TEM images as shown in Fig. 2(a) for the wurtzite structure and Fig. 2(b) for the zinc-blende structure. An example of GPA used for the positioning of the CdTe markers along the NWs is presented in Fig. 2(c). Fig. 3(a) shows the growth sequence. In Fig. 3(b), the horizontal axis is the total amount of ZnTe sent before a CdTe marker is inserted (position indicated by the red segments), normalized to 1 for the complete growth sequence. Data plotted at complete growth sequence display the visible length of the NW. It should be kept in mind that a 2D layer masks the bottom of the NWs: from a SEM image of a cleavage 


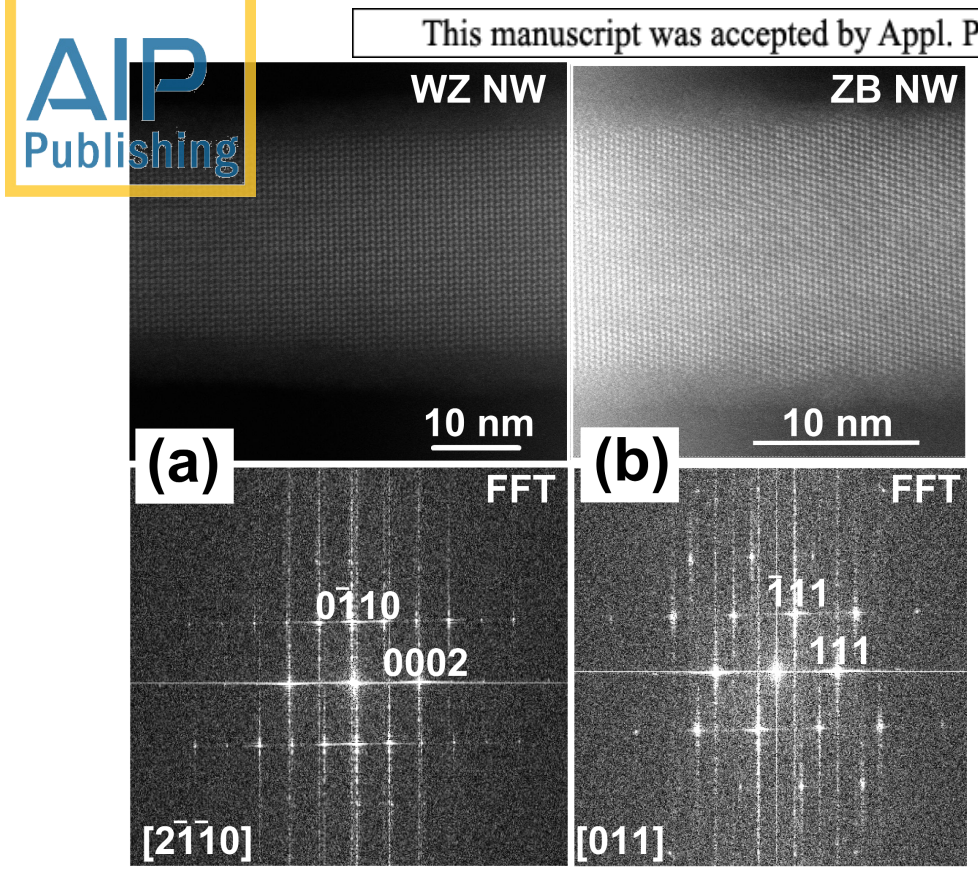

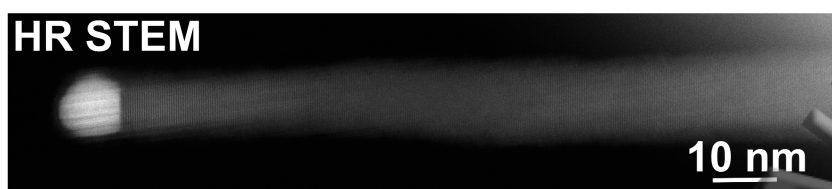

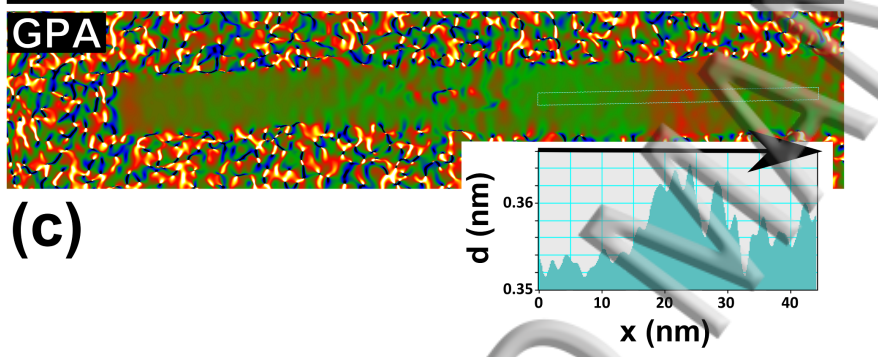

FIG. 2. High resolution TEM images and corresponding Fourier Transform of (a) a wurtzite NW, (b) a zinc-blende NW. (c) Structural analysis of a ZnTe NW with CdTe markers, containing: the HR-STEM image, the corresponding GPA map of the growth plane and the interplanar distance extracted from the boxed region in the GPA image. $0.352 \mathrm{~nm}$ corresponds to the lattice parameter of relaxed ZnTe.

plane, we estimate this $2 \mathrm{D}$ layer to be $\sim 100 \mathrm{~nm}$ thick, with $\sim 70 \mathrm{~nm}$ of $\mathrm{ZnTe}$, in agreement with the growth sequence and previous calibrations. Data at values $<1$ display the position of each CdTe marker, with respect to the NW tip, as determined by the GPA analysis of the STEM images (6 NWS) or by EDX (2 NWs). These NWs can be identified as wurtzite or zinc-blende from their morphology, cone-shaped or cylinder-shaped with a pyramid at the basis; the crystal structure was directly confirmed on the STEM high-resolution images for 2 zinc-blende and 2 wurtzite NWs. Two distinct features appear from these raw data, without further analysis: (1) the data of all NWs converge to a single point and
(2) they are grouped in two families corresponding to the zinc-blende NWS and the wurtzite NWs, respectively. A direct, qualitative interpretation of these two features is that (1) the presence of a convergence point suggests an identical start of all NWs, its position along the vertical axis denoting the thickness of the $2 \mathrm{D}$ layer (i.e., the total NW length should be measured from this position) and (2) the growth rate of wurtzite NWs is smaller than that of zinc-blende NWs, with a small dispersion within each family.

A more detailed analysis can be obtained using the diffusion driven growth model of Ref. 9, as shown in Fig. 3(c)-(d). We consider only the zinc-blende NWs. The wurtzite NWs can be modeled as well, assuming vanishing lateral regrowth. However less information can be gained in this case. Fig. 3(c) was deduced from the raw data in Fig. 3(b), as follows. The horizontal axis is the thickness of the 2D layer when taking into account the $\mathrm{ZnTe}$ contribution only. The small height of each CdTe marker was measured and subtracted from the raw data. Finally, knowing the position of each CdTe marker and the thickness of the 2D layer at the time the marker was grown, we can calculate the value of the NW length at this time. This is plotted in Fig. 3(c), and Fig. 3(d) is a reminder of the data of Ref. 9 for the sake of comparison. The solid lines are obtained using the diffusion-driven growth model of Ref. 9. The most important fitting parameter is the ratio $\frac{\lambda_{N W}}{2 R_{\text {tip }}}$ between $\lambda_{N W}$, the diffusion length on the NW sidewalls, and $R_{t i p}$, the contact radius between the gold nanoparticle and the NW tip: it impacts the growth rate, i.e., the slope in Fig. 3(c)-(d). A second important feature is the position along the horizontal axis: it is determined by the value of the incubation time (expressed in 2D layer thickness, $\left.h_{0}\right)$, although it can be influenced by the value of the diffusion length on the substrate, $\lambda_{s}$ (which changes the initial growth rate).

The behavior of the two samples is strikingly different. In the case of vacuum dewetting, Fig. 3(d), the radius $R_{t i p}$ was measured on the STEM high-resolution image of each NW, and the fit involves the same values of the diffusion length, $\lambda_{N W}=78 \mathrm{~nm}$, but markedly different values of the incubation time, $h_{0}=105 \mathrm{~nm}$ for the shortest and $h_{0}=28 \mathrm{~nm}$ for the longest NW. In the case of Zn-dewetting, Fig. 3(c), the values of $\frac{\lambda_{N W}}{2 R_{t i p}}$ are not significantly affected and show a weak dispersion from NW to NW (but $R_{\text {tip }}$ cannot be measured since the last sequence is a CdTe insertion, which significantly alters the nanoparticle shape). Fig. 3(c) thus confirms that the most dramatic effect of $\mathrm{Zn}$-dewetting is the suppression of the dispersion in the incubation time.

A statistical analysis of pure ZnTe NWs, including a sample with a longer growth time, brings complementary information on the remaining length dispersion and its origin. This is done in Fig. 4 where we again consider only the zinc-blende NWs of the two samples forming series C. Fig 4(a) displays two NWs which were 

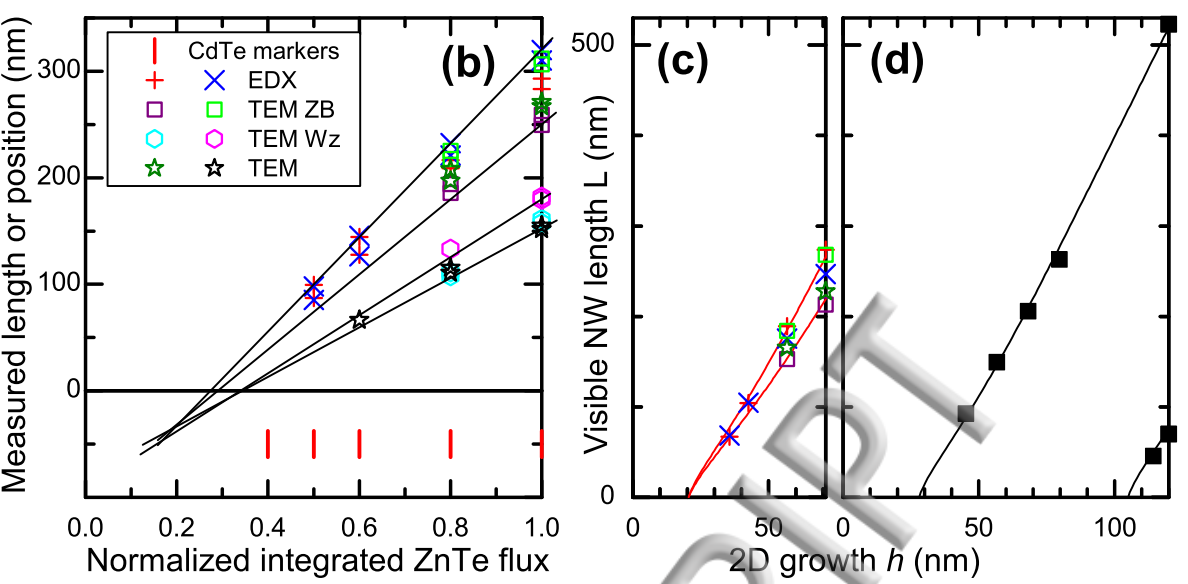

FIG. 3. (a) Growth sequence; (b) raw data on the visible length of the NWs and the position of the CdTe markers, for different NWs, as measured by STEM and EDX, with respect to the amount of ZnTe sent at the time the marker was inserted (normalized to the whole growth sequence). For each marker, the position of the two interfaces (CdTe on ZnTe and ZnTe on CdTe) are indicated (note the limited growth of CdTe at this temperature ${ }^{17}$ ). The red segments indicate the positions of the markers. The black solid lines are eye-guides. (c) Same data analyzed along the diffusion-driven growth model of Ref. 9: visible ZnTe NW length $v$ s. the thickness of the ZnTe 2D layer, both at the time the marker was inserted. Solid lines are calculated with $h_{0}=20 \mathrm{~nm}$, $2 R_{\text {tip }}=7.8 \mathrm{~nm}$ and $9.3 \mathrm{~nm}$, respectively, $\lambda_{N W}=78 \mathrm{~nm}$ and $\lambda_{s}=18 \mathrm{~nm}$. (d) Reprinted from J. Appl. Phys. 119,164303 (2016). Solid lines are calculated with $h_{0}=28 \mathrm{~nm}$ and $2 R_{\text {tip }}=7.7 \mathrm{~nm}$, and $h_{0}=105 \mathrm{~nm}$ and $2 R_{\text {tip }}=7.9 \mathrm{~nm}$, respectively, $\lambda_{N W}=78 \mathrm{~nm}$ and $\lambda_{\mathrm{s}}=18 \mathrm{~nm}$.

included in the statistics: indeed we observe NWs with a nanoparticle at the tip forming a half-sphere (similar to the shape observed after dewetting), and other NWs with a nanoparticle forming a quasi-full-sphere. For the same volume of the nanoparticle, the diameter of the full sphere is slightly smaller (by a factor $\sqrt[3]{2}$ ), and the tip contact radius $R_{\text {tip }}$ is much smaller. As the growth rate depends on $R_{\text {tip }}$, we thus expect an influence of both the nanoparticle volume and the nanoparticle shape.

In the frame of the diffusion-driven model ${ }^{9}$, if the tip contact diameter is maintained during the whole process, a plot of the ratio between the base diameter and the tip diameter (i.e., the radial growth normalized to the initial diameter), as a function of the NW length, should follow a single curve, independent of the nanoparticle radius and possible incubation time (see Equ. 2 in Ref. 9). If the nanoparticle keeps a half-sphere shape over the whole growth process, the relevant radius $R_{\text {tip }}$ is simply the nanoparticle radius. Fig. $4(\mathrm{~b})$ displays such a plot for about one hundred NWs of series $C$, with both half-sphere nanoparticles and quasifull-sphere nanoparticles, as indicated. The average gold catalyst dimension with its standard deviation is $13 \pm 3 \mathrm{~nm}$ for the 40 minutes sample. The solid line is calculated for $\lambda_{N W}=65 \mathrm{~nm}$ and $\lambda_{s}=20 \mathrm{~nm}$, the most significant parameter being actually $\lambda_{s}^{2} / \lambda_{N W}^{3}$ : it sets the upper limit of the distribution of experimental data. If we now assume that the nanowire shape is full sphere, the tip radius is smaller than the nanoparticle radius, and the calculated curve has to be multiplied by the corresponding reduction factor, $R_{t i p} / R_{N P}$. In Fig. $4(\mathrm{~b})$, the curve with $R_{t i p} / R_{N P}=0.6$ sets the lower limit. We find that all observed NW lengths are between the limits expected for the full sphere and half sphere catalyst particle respectively (black and red lines). However, no clear separation is visible between the NWs having a full sphere, and those having a half sphere catalyst particle. It appears therefore that catalyst particles can change shape during growth, or alternate between a full and a half sphere particle. This effect of the two possible shapes of the nanoparticle was already noticed in Ref. 9 and it is still present.

In order to evidence the possible influence of the nanoparticle size, Fig. 4(c) displays the NW length as a function of the nanoparticle diameter evaluated on the SEM images. In principle, the relevant radius here should be the tip radius $R_{\text {tip }}$. However we have here only a rather crude evaluation of the radius, due to a moderate resolution, and to the presence of an amorphous layer which is probably a mix of oxides and redeposition. The lines are drawn using Eq. 1 of Ref. 9, with the same values of the diffusion lengths as in Fig. 4(b), and a common incubation time $h_{0}=20 \mathrm{~nm}$ (and no incubation time for the dotted line). The effect of the nanoparticle size is present, although not dominant; it was completely hidden by the effect of the incubation time in Ref. 9.

To sum up, the residual dispersion of the zinc-blende NWs is due to both the random change of shape of the nanoparticle (as evidenced by the images in Fig. 4(a) 


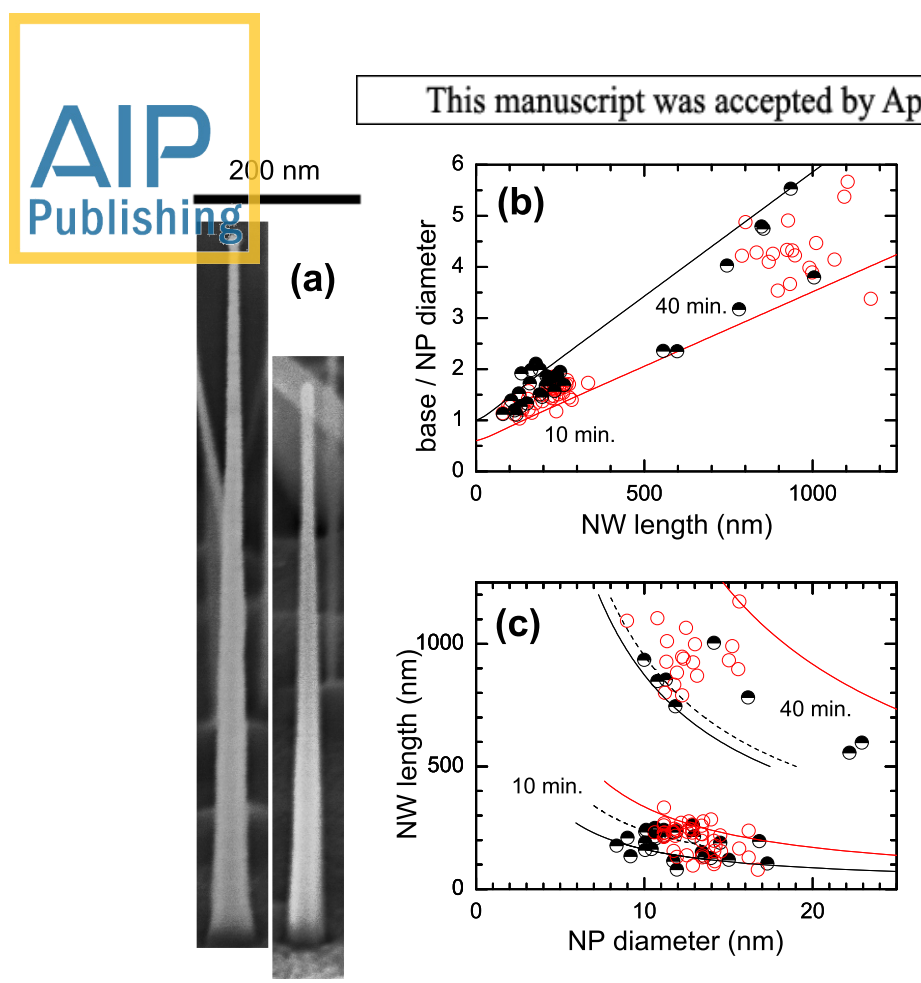

with nanoparticles of different shapes, sizes and orientation. We may expect similar features in the case of $\mathrm{ZnTe}$, with possible effects on the first steps of growth of the NWs.

To conclude, applying a $\mathrm{Zn}$ flux during the dewetting of gold nanoparticles has a positive effect on the growth of ZnTe NWs, with a quasi suppression of the dispersion in the incubation time, and no significant change in the subsequent growth of the ZnTe NW. A main consequence is a reduction of the length dispersion from more than 10 to about 2 . This residual dispersion is related to two effects: a (random) change of the nanoparticle shape during the growth, and a dispersion in the nanoparticle size.

\section{ACKNOWLEDGMENTS}

This work was supported by the French National Research Agency under Contracts ANR-11-BS10013, ANR-15-CE24-0029, ANR-12-JS10-0002 and labex LANEF ANR-10-LABX-51- 01. We benefited from the access to the technological Nano Characterization plat-

FIG. 4. (a) $65^{\circ}$ tilted SEM images of cone-shaped NWs from series $\mathrm{C}$, featuring the two characteristic shapes of the nanoparticle, almost full-sphere and half-sphere; (b) ratio of the base diameter to nanoparticle diameter, as a function of the NW length. (c) NW length $v$ s. the nanoparticle diameter. The half-filled symbols identify the NWs with an half-sphere nanoparticle and the open symbols the full-sphere. The lines are calculated in the diffusion-driven model of Ref. 9, assuming half-sphere nanoparticles throughout the growth (black solid lines), or full-sphere with a tip radius 0.6 times the nanoparticle radius (red solid line) with an incubation time $h_{0}=20 \mathrm{~nm}$. The dotted line is calculated with no incubation time.

and by the corresponding shifted clouds in Fig. 4 (b) and (c)), and the dispersion of the nanoparticle size (as evidenced in Fig. 4(c)).

The diffusion-driven model, applied in Fig. 3(c) and Fig. (4), evidences that the presence of a $\mathrm{Zn}$ flux during the dewetting dramatically influences the first step of growth (incubation time) and not the characteristics of the growth once started. Note that this model is purely phenomenological; for instance, the diffusion lengths are not attributed to $\mathrm{Zn}$ or $\mathrm{Te}$, and they are assumed to be constant throughout the growth. A part of the remaining incubation time could be due to a very low value of $\lambda_{s}$ at the beginning of growth. One motivation to use VSS instead of VLS, is the small amount of constituents of the NWs which are expected to be present in the gold solid nanoparticles, as compared to gold liquid droplets. A new parameter however emerges in the case of VSS: the crystal structure of the nanoparticle, and its orientation with respect to the substrate and to the NWs. A complete study of gold nanoparticles on a $\mathrm{Si}(111)$ substrate has revealed ${ }^{19}$ a very rich diagram, form of CEA-Minatec.

${ }^{1}$ N. P. Dasgupta, J. Sun, C. Liu, S. Brittman, S. C. Andrews, J. Lim, H. Gao, R. Yan, and P. Yang, Adv. Mater. 26, 2137 (2014).

${ }^{2}$ Y.-M. Niquet and D. C. Mojica, Phys. Rev. B 77, 115316 (2008).

${ }^{3}$ M. Zieliński, Phys. Rev. B 88, 115424 (2013).

${ }^{4}$ Y. Huo, B. Witek, S. Kumar, J. Cardenas, J. Zhang, N. Akopian, R. Singh, E. Zallo, R. Grifone, and D. Kriegner, Nature Phys. 10, 46 (2014).

${ }^{5}$ M. Jeannin, A. Artioli, P. Rueda-Fonseca, E. Bellet-Amalric, K. Kheng, R. André, S. Tatarenko, J. Cibert, D. Ferrand, and G. Nogues, Phys. Rev. B 95, 035305 (2017).

${ }^{6}$ M. Szymura, P. Wojnar, u. Kłopotowski, J. Suffczyński, M. Goryca, T. Smoleński, P. Kossacki, W. Zaleszczyk, T. Wojciechowski, G. Karczewski, T. Wojtowicz, and J. Kossut, Nano Lett. 15, 1972 (2015).

${ }^{7}$ C.-Y. Wen, M. C. Reuter, J. Bruley, J. Tersoff, S. Kodambaka, E. A. Stach, and F. M. Ross, Science 326, 1247 (2009).

${ }^{8}$ P. Rueda-Fonseca, E. Bellet-Amalric, R. Vigliaturo, M. den Hertog, Y. Genuist, R. André, E. Robin, A. Artioli, P. Stepanov, D. Ferrand, K. Kheng, S. Tatarenko, and J. Cibert, Nano Lett. 14, 1877 (2014).

${ }^{9}$ P. Rueda-Fonseca, M. Orrù, E. Bellet-Amalric, E. Robin, M. Den Hertog, Y. Genuist, R. André, S. Tatarenko, and J. Cibert, J. Appl. Phys. 119, 164303 (2016).

${ }^{10}$ S. Breuer, M. Hilse, A. Trampert, L. Geelhaar, and H. Riechert, Phys. Rev. B 82, 075406 (2010).

${ }^{11}$ F. Dhalluin, T. Baron, P. Ferret, B. Salem, P. Gentile, and J.-C. Harmand, Appl. Phys. Lett. 96, 133109 (2010).

${ }^{12}$ C. O'Regan, S. Biswas, C. O'Kelly, S. J. Jung, J. J. Boland, N. Petkov, and J. D. Holmes, Chem. Mat. 25, 3096 (2013).

${ }^{13}$ P. Sahoo, S. Dhara, S. Amirthapandian, and M. Kamruddin, J. Mater. Chem. C 1, 7237 (2013).

${ }^{14}$ F. Bastiman, H. Küpers, C. Somaschini, and L. Geelhaar, Nanotechnology 27, 095601 (2016).

${ }^{15} \mathrm{M}$. Hÿtch, E. Snoeck, and R. Kilaas, Ultramicroscopy 74, 131 (1998).

${ }^{16} \mathrm{~J}$. Rouvière and E. Sarigiannidou, Ultramicroscopy 106, 1 (2005).

${ }^{17}$ P. Wojnar, J. Płachta, S. Kret, A. Kaleta, W. Zaleszczyk, M. Szymura, M. Wiater, L. T. Baczewski, A. Pietruczik, G. Karczewski, T. Wojtowicz, and J. Kossut, Nanotechnology 28, 045207 (2017).

${ }^{18}$ F. Glas, J.-C. Harmand, and G. Patriarche, Phys. Rev. Lett. 104, 135501 (2010).

${ }^{19}$ R. Daudin, T. Nogaret, T. U. Schülli, N. Jakse, A. Pasturel, and G. Renaud, Phys. Rev. B 86, 094103 (2012). 

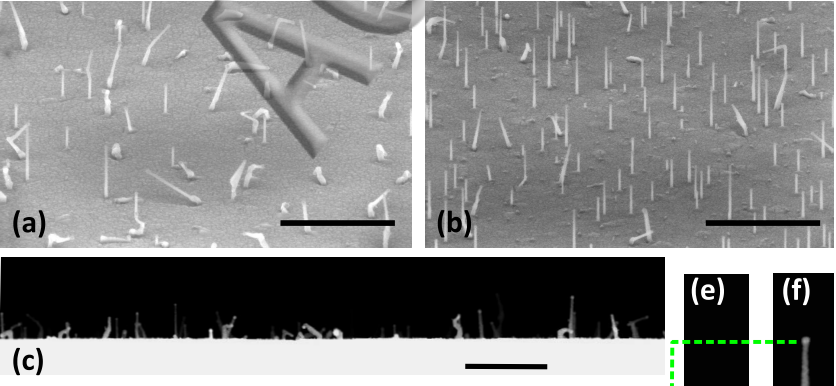

(d)

(e)

(f)

(c)

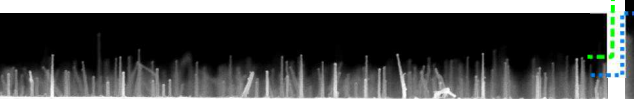

$500 \mathrm{~nm}$

$-50 \mathrm{~nm}$ 
(a)
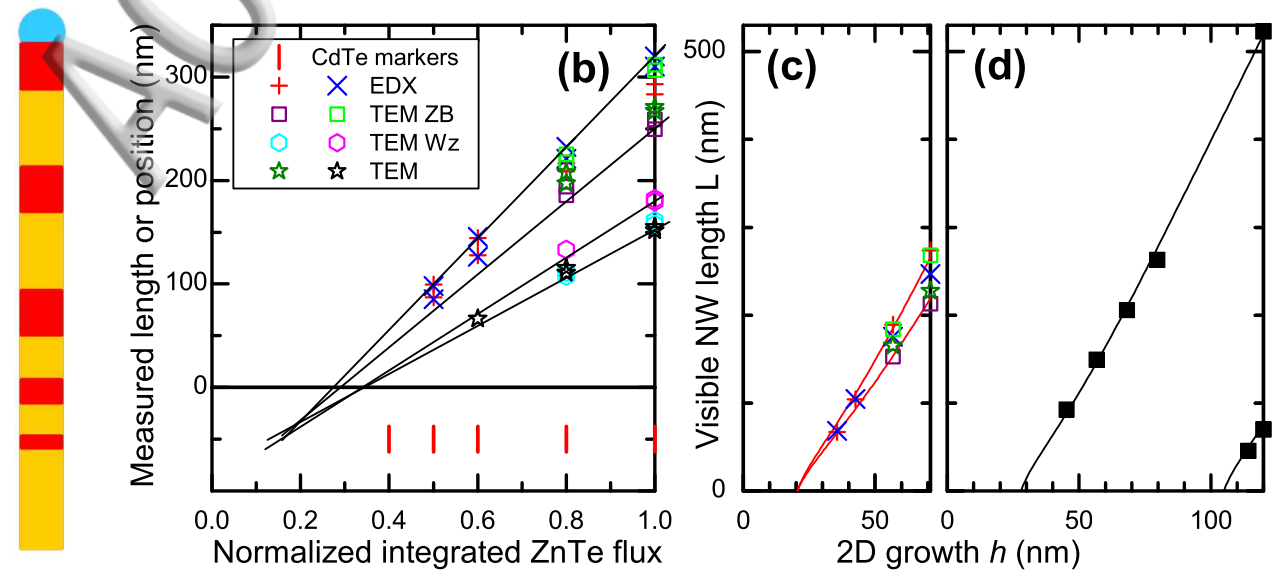

$\mathrm{ZnTe}$

$\mathrm{CdTe}$ 
$200 \mathrm{~nm}$

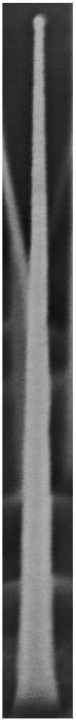

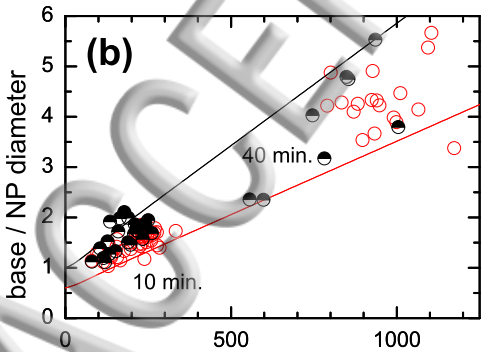

NW length (nm)

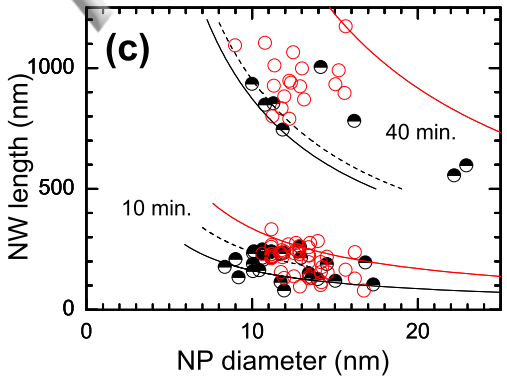

